\title{
Modeling of Seamless Vertical Handover Mechanism using Demanded Resource Identification \& Mapping
}

\author{
S. B. Kumbalavati ${ }^{1,2}$ \\ ${ }^{1}$ Department of Electronics and Communication Engineering, Jain University, Karnataka, India \\ E-mail: sbkumbalavati@gmail.com \\ J. D. Mallapur ${ }^{2}$ \\ ${ }^{2}$ Department of Electronics and Communication Engineering, Basaveshwar Engineering College, \\ Bagalkot, Karnataka, India \\ E-mail: bdmallapur@yahoo.co.in
}

Received: 14 April 2018; Accepted: 20 July 2018; Published: 08 August 2018

\begin{abstract}
One of the prominent challenges for offering seamless communication system while performing vertical handover in heterogeneous network is to relay the communication without identifying the accurate demands of the resources as well as quality of services for the newly moved node. After reviewing the existing literatures, it was found that there is a potential research gap in addressing this problem of seamless vertical handover. Therefore, the proposed manuscript addresses this problem by introducing a novel analytical model which is capable of formulating a precise decision for controlling the selection /dropping of the data packets on the basis of dynamic state of the network condition. The proposed system contributes faster processing by arbitrarily selecting the packets to be forwarded with a very unique and simple resource management. The study outcome of proposed system highlights an increased throughput and reduced length of queue along with better fairness control to offer seamless vertical handover.
\end{abstract}

Index Terms-Handover, vertical handover, Resource, Quality of Service, Seamless Heterogeneous Network.

\section{INTRODUCTION}

There has been a series of research work towards improving the performance of communication during vertical handovers [1,2]; however, the solution offered by the existing research approaches are highly scattered. A heterogeneous network usually considers of discrete group of networks with different forms of access technologies e.g. WLAN, CDMA, WiMAX, LTE, IEEE 802.XX family of standards with unique form of research challenges $[3,4,5]$. While performing vertical handover, it is essential to ensure less occurrences of call drop however it is yet an unsolved problem. At present, existing research work towards vertical handover process is always carried out considering only two forms of networks say WLAN and CDMA network, WiMAX and
Mesh Network, WLAN and 4G network, etc. Such forms of research case studies have a very limited list of network characteristics, which is not so challenging to address. For an example, the research approaches that states migration of node from WLAN to CDMA work efficiently may not work if CDMA is changed to $4 \mathrm{G}$ or something else. The change of access technologies has significant impact on the vertical handover process and this problem is very much overlooked $[6,7,8]$. At the same time, identification of a precise amount of resource requirement of a node is extremely important for a mobile station moving to a new foreign network without which the service relaying will be incompatible. A significant research gap is found in existing literature where none of the vertical handover management has been ever modeled by considering the constraints associated with capabilities of the mobile node $[9,10]$. Usually, a mobile node has limited energy, limited computational capabilities, as well as less memory; therefore, it is quite computationally challenging task to maintain scheduling-based information within a mobile node [11]. This paper presents a technique where resource identification and quality of service issued for carrying of effective vertical handover in presence of heterogeneous network.

The organization of the paper is as follows: Section A discusses about the existing literatures where different techniques are discussed for detection schemes used in power transmission lines followed by discussion of research problems in Section B and proposed solution in C. Section II discusses about algorithm implementation followed by discussion of result analysis in Section III. Finally, the conclusive remarks are provided in Section IV.

\section{A. Related Works}

This section discusses about the existing methodologies evolved in literatures towards addressing problems associated with resource management and quality of service during vertical handover. The issues of resource utilization were addressed by Ali et al [12] by 
developing a cooperative model of management of radio resources and vertical handover. Study considering resource management was carried out by Suga and Tafazolli [13] where the authors have used coding-based implementation scheme to confirm more precision decision towards vertical handover. Remnant resource scale is used as an indicator factor for formulating a method of vertical handover as seen in the work of Wang and Kao [14]. Improvement of query mechanism for resources during vertical handover was investigated by Zahran and Sreenan [15] considering architecture designed using broker-based scheme for addressing delay problems too. Adaptive approach for constructing an efficient vertical handover system was introduced by Guo and $\mathrm{Li}$ [16] on the basis of the cost function. Further study towards optimization was carried out by Liu et al. [17] which basically performs selection of optimal network for minimizing computational load using software defined network. Such form of network and its feasibility was also investigated by Nyugen-Duc and Kamioka [18] considering real-time testbed of personal area network. Vuchkovska and Jakomosi [19] have addressed the problems of delay during vertical handover considering the case study of specific networking technologies e.g. WLAN and WiMAX.

Load balancing-based approach was seen in the work of Gao et al. [20] for minimizing ping-pong effect on 4G networks. Similar approach was introduced by many authors e.g. Manfredi [21]; however, they misses considering vertical handover problems. Load-balancing scheme is also investigated by Rym and Afif [22] by considering mobilityfactors of the user for minimizing delay. Delay problem was also addressed by Yadollahi et al. [23], the approach is very similar to that of Gao et al.[20].Consideration of diverse criteria for performing the vertical handover was investigated by Mahardhika et al. [24] on the basis of traffic intensity. Such forms of traffic introduce higher number of delay which is also found less emphasized in existing system. Researchers e.g. Rajule and Ambudkar [25] have addressed this problem using Markov process where delay is considered to essential criteria for performing an effective vertical handover. Usage of intelligence-based approach was another frequently used technique to form efficient decision for vertical handover. The study carried out by Fachtali et al. [26] has used ant colony optimization for carrying out optimization. Adoption of machine-learning based approach was seen in the work of Kunarak [27] by addressing the problem associated with channel allocation. Similar adaptive-based approach was also found to be assisting modeling of vertical handover using fuzzy logic system. Usage of fuzzy logic was reported for assisting in effective handover in heterogeneous network as seen in work of Shanmugam [28] addressing some of the hypothetical factors of signals. Adoption of bioinspired techniques has been seen in the work of Tian et al. [29] to ensure adherence to the quality of service standard in presence of heterogeneous networks. A predictive approach for handover for assisting seamless communication by the mobile relay was discussed by Ali et al. [30] considering a case study of railways. The next section outlines the research problems.

\section{B. Research Problem}

The significant research problems are as follows:

- Existing research approaches doesn't emphasize on relaying the vertical handover service with respect to the dynamic demands in the foreign network for the newly moved mobile station.

- Memory management of the mobile station is highly overlooked when it comes to vertical handover in heterogeneous network.

- The existing decision for performing vertical handover involves too much complex steps and have narrowed coverage of access technologies

- There is a lack of emphasis of identification of precise channel capacity as well as quality of service parameters while performing vertical handovers.

Therefore, the problem statement of the proposed study can be stated as "Developing a computational model for relaying vertical handover service by dynamically identifying the capabilities of the node in heterogeneous network is challenging one." The next section discusses about proposed solution.

\section{Proposed Methodology}

The proposed research work is carried out considering analytical research methodology using simplified mathematical modeling. The adopted architecture of the proposed study has been highlighted in Fig.1. The research challenge that it addresses is:- when a new mobile station joins a new foreign network, it is absolutely not necessary that vertical handover will take place effectively. There are various intrinsic and extrinsic factors that will degrade the vertical handover performance especially if there are large numbers of heterogeneity in the network. The probable scenarios are i) network device in foreign network is of higher capacity and memory of node is less in such case it will encounter buffer overflow problem, ii) if the network device in foreign network is of lower capacity than it could possibly introduce delay and could also lead to bottleneck condition. This form of incompatibility between the network device and mobile station will not allow seamless vertical handover. The root cause of this problem is lack of identification of effective need of resources as well as ignorance of quality of service parameters. The proposed system addresses this problem by introducing a new actor called as extracting attribute which will be responsible for controlling the data to be forwarded by the network device.

Following are the steps of operation that are carried out in proposed system viz.

- The mobile station forwards a request req to the network device of foreign agent to have an accessibility for its resources that is originally 
retained in its home network. Apart from synching with base station of home network, the network device will perform some addition task, unlike conventional vertical handover.

- The network device will compute the difference forms of extraction attributes as well as saturation state of memory and maintain all this information in a master matrix (within its memory shared by other network devices too). This process allows the network to obtain dynamic information about the incoming traffic which assists in formulating a decision about taking necessary steps for serving the mobile station for its generated request.

- The next step will be to perform a comparison between the existing traffic conditions with the predefined condition of the state during vertical handover. This predefined condition is specific for different forms of the access technologies and therefore it offers highest frequency of updates while performing each event of vertical handover.

- The network device than computes the updated values of extraction attribute as well as saturation state of the memory, based on which, the network device will allocate discrete channel capacity to the requesting node. The construction of this logic was carried out using probability theory.

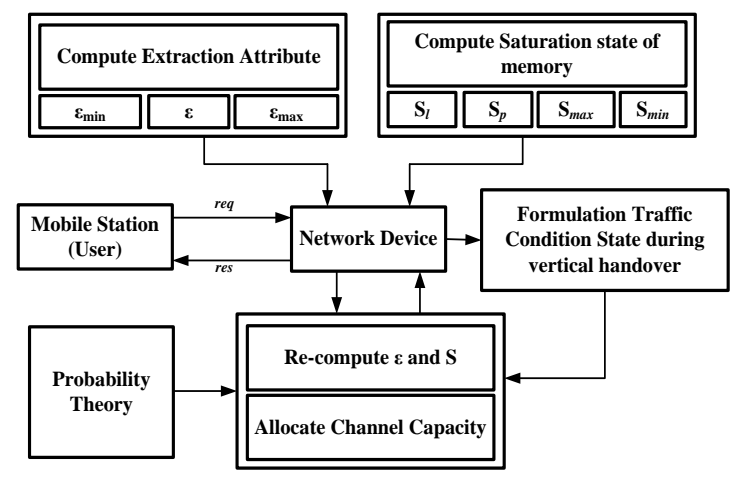

Fig.1. Proposed Architecture

Based on the response res of network device, the mobile station either drops the complete data or drops partial data or doesn't drop at all and forward the data to the network device which further forwards it to the destination node. The next section illustrates the algorithm that is constructed to achieve the above stated research methodology.

\section{ALGORITHM IMPLEMENTATION}

The core motive of this algorithm is to control the flow of data during vertical handover in presence of heterogeneous network. The prime idea is to extract different data packets from the memory (output) of the node followed by comparison with the incoming traffic network in order to streamline and balance the flow. The secondary motive of this algorithm design is to offer an efficient allocation of quality of service parameters in order to assists in optimal communication during the vertical handover mechanism. This is a significant novelty feature that has never being attempted in literature. Hence, extracting attribute is one of the novel parameter that assists in measuring the capability of the node along in order to maintain nearly similar form of quality of service performance while performing vertical handover. The steps involved in this novel algorithm for computing extracting attribute are as follows:

\section{Algorithm for Computing Extracting Attribute in Foreign Network}

Input: $\varepsilon$ (Extraction Attribute), $p$ (priority), $S_{\mathrm{p}}$ (Saturation

State of memory)

Output: $\varepsilon_{\text {final }}($ Final Extraction Attribute)

\section{Start}

1. compute $\varepsilon, \operatorname{Pr}(\varepsilon)$

2. For $i=0:$ data $(\mathrm{p})$

3. $\mathrm{N}_{\mathrm{d}} \rightarrow \operatorname{eval}\left(\mathrm{S}_{\mathrm{p}}\right)$ //actually $\mathrm{Nd}$ is router

4. If $\mathrm{S}_{\mathrm{p}}<\mathrm{S}_{\mathrm{th}}$

5. $\quad \varepsilon_{\text {final }} \rightarrow 0$

6. Elseif $\mathrm{S}_{\min }<\mathrm{S}_{\mathrm{p}}>\mathrm{S}_{\mathrm{th}}$

7. $\varepsilon_{\text {final }}=\left|\varepsilon-\varepsilon_{\min }\right| \max$

8. Elseif $\mathrm{S}_{\min }<\mathrm{S}_{\mathrm{p}}<\mathrm{S}_{\max }$

9. $\varepsilon_{\text {final }}$

10. Elseif $_{\mathrm{p}}>\mathrm{S}_{\max }$

11. $\varepsilon_{\text {final }} \rightarrow \varepsilon+\theta$. $\varepsilon_{\text {max }}$

12. End

13. End

End

In order to maintain better practicality in the proposed concept, we define $\varepsilon$ (Extraction Attribute) as the cumulative cardinality of data that should be extracted from the memory allocated for outgoing traffic within a mobile node. The dominant contribution of this algorithm is that this computation of $\varepsilon$ (Extraction Attribute) is carried out by exponential increasing step till the size of the queue is found to be maximum while it minimize to one step when the size of the queue is found to be minimal. Hence, it behaves in the form of opportunistic communication mechanism. The computation of $\varepsilon$ (Extraction Attribute) is carried out by summing both integer part $i$ and floating part $f$ of $\varepsilon$ (Extraction Attribute) (Line-1). The system then computes the probability $\operatorname{Pr}$ as follows,

$$
\operatorname{Pr}=\left\{\begin{array}{cc}
1-j & i \\
j & i+1
\end{array}\right.
$$

The above expression (1) will mean that probability $\mathrm{Pr}$ of extracting attribute will be (1-j) for extracting $i$ number of data while probability $\operatorname{Pr}$ will be $j$ for extracting $(i+1)$ number of data (Line-1). The implementation of the study considers that there is presence of different forms of network devices $N d$ based on the different types of networks available during vertical handover. In this process, the root $\mathrm{Nd}$ will forward its neighborhood traffic statistics based on which the communicating node will have to drop its specific number of data (if required in 
case of bottleneck situation). Fig.2 highlights the mechanism of allocation of the extraction attribute $\varepsilon$ residing within the memory. The proposed algorithm also maintains a significance level $p$ of the data represented as $\operatorname{data}(p)$ (Line-2). Once the incoming traffic arrives the network devices performs a preliminary check of the saturation state of the memory represented as $S_{\mathrm{p}}$ that is basically utilized for comparing the already defined cutoff value. In case the level of saturation state of the memory $S_{\mathrm{p}}$ is found less than the minimum cut-off value $S_{\text {th }}$, it will mean that the amount of channel capacity is nearly similar to the both the network device as well as the requesting node in the new network during vertical network. Hence, in situation, there is no event of data to be dropped under such circumstances (Line-4) and the extraction attribute is made to be reconfigured as 0 . It will mean that there is no requirement of extracting any data from the memory of the node in the foreign network. This is one of the preliminary conditions where the algorithm has to decide when not to drop the data.

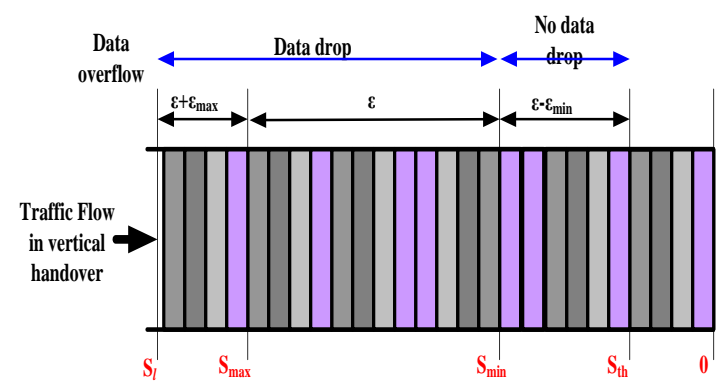

Fig.2. Data Scheduling process in Proposed System

If the situation arise in such way that saturation state of the memory $\mathrm{Sp}$ is found to be numerically more than the minimum allotted value of $\mathrm{S}$ as well as it is also found equally to be higher than the cut-off value of saturation state $S$ (Line-6), than the algorithm performs a simpler computation of the final extraction attribute $\varepsilon_{\text {final }}$. In such case, the extracting attribute could be possibly more and could lead to odd environment of vertical handover mechanism leading to bottleneck situation at some point of time. This possibility is avoided by minimizing the extraction attribute by subtracting the total value of extraction attribute $\varepsilon$ with minimal value of extraction attribute $\varepsilon_{\text {min. }}$. The algorithm performs an evaluation of next condition where there is possibility of saturation state of the memory $S_{\mathrm{p}}$ to be less than higher value of it i.e. $S_{\max }$ as well as it could be found more than the minimal value of saturation state of memory $S_{\min }$ (Line-8). In such condition, actual value of extraction attribute is not subjected to any form of alteration and is considered as the final value itself (Line-9). Finally, when the saturation state of memory $S_{p}$ is found to be more than higher value of cut-off level (Line-10), the network device will have to undergo maximum load of the incoming traffic in order to balance the resource demands of the requestor node in foreign network. It does so by increasing the extraction attribute by summing up the extraction attribute with maximum extraction attribute (Line-11). A new variable $\theta$ is used for representing incremental operator and it is empirically expressed as,

$$
\theta=\left|\frac{f(s)}{g(s)}\right|
$$

In the above expression (2), the two functions $f(\mathrm{~S})$ and $g(\mathrm{~s})$ represents $\left(S_{\mathrm{p}}-S_{\max }\right)$ and $\left(S_{\max }-S_{\min }\right)$ respectively. The algorithm will also consider that when the saturation state of the memory $S_{\mathrm{p}}$ is found to be more than the permissible bound $S_{1}$ than the incoming traffic with data will be subjected to be dropped with highest probability of 1 . There are two process of extraction carried out in proposed system during vertical handover. In the first process, the data is chosen by the network device which mainly check the index of the traffic flow say $\delta_{1}$ and when it flow is found to be similar corresponding ot the index of incoming packet $\delta_{2}$ than the network device drops such network flow using the extraction attribute of $\varepsilon$. The next step is to check the level of significance in both the traffic flows. Considering a cut-off level of $H_{\mathrm{p}}$, when the traffic situation during vertical handover shows that cardinality of data with significance level of $p$ is found to be more than $H_{\mathrm{p}}$, than the surplus data are extracted from the pipeline of communication and is instantly subjected to be dropped. This is required to perform allocation of precise channel capacity based on discrete level of significance value of data. Hence, the allocated channel capacity can be said to be equivalent to $H_{\mathrm{p}}$ and applying probability, the total possible value of $H_{\mathrm{p}}$ is always 1 from entire ranges of significance level. The empirical expression to represent this condition is,

$$
\sum_{p=1}^{p} H_{p}=1
$$

Therefore, a closer look into the present state of vertical handover process will show that the extraction attribute maximizes with increased momentum when the traffic flow meet a bottleneck condition while the process of extraction reduces upon reduction of bottleneck condition. This is an ideal condition of vertical handover in heterogeneous network where is higher probability of dynamic and bursty traffic situation. At the same time, it also significant saves overflow of memory. Hence, the proposed system shows a good capability to perform an effective resource management as well as retain better amount of quality of service performance. The next section discusses about the outcome obtained from the proposed study.

\section{RESUlt ANALYSIS}

As the proposed system focuses on enhancing the performance of the vertical handover mechanism by efficiently identifying the exact amount of resource resourced dynamically in presence of heterogeneous network. The simulation is carried out in MATLAB 
considering mean size of data to be 1000 bytes with a memory limit of 450 packets. The performance parameters considered are throughput and queue length. The study also uses a new performance parameters called as Fairness Index F which is computed by summation of throughput divided by product of throughput and number of traffic flows. The study also considers performing a comparative outcome with the related work carried out by Mahardhika et al. [24] which uses multiple metrics in order to carry out vertical handover. The study is highly cited and bears common objective so it is considered for performing benchmarking with the existing system.

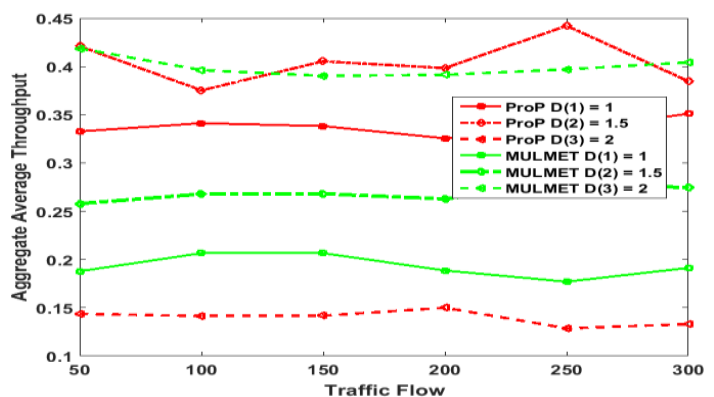

Fig.3. Comparative analysis of Throughput on three degrees of Significance

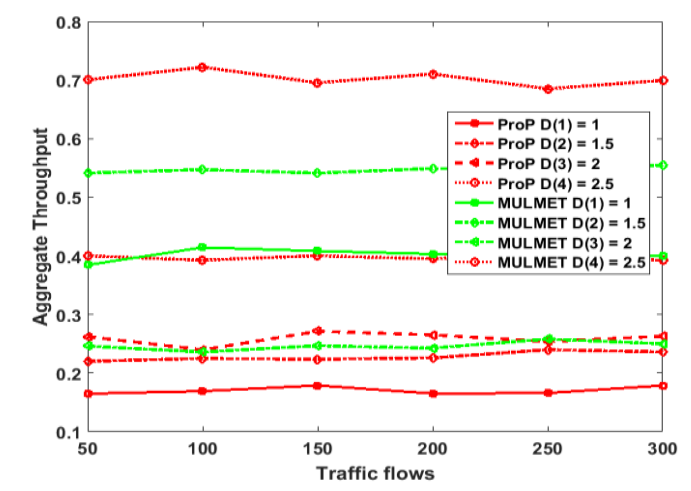

Fig.4. Comparative analysis of Throughput on four degrees of Significance

For an effective analysis, the degree of significance is considered as a weighing coefficient for representing the p significance while performing simulations. Considering the probability scale, it is found that proposed system offers increasing performance of the aggregate throughput with an increasing flow of the traffic in contrast to the existing MULMET system. Increase of degree of significance has been found to show increase of an aggregated throughput and the overall performance is quite uniform for increase of traffic flow. The performance of the MULMET is degraded compared to proposed PRoP because of highly inclusion of large number of conditional factors which are highly recursive in order with lesser updates. This results in minimization of throughput. Moreover, MULMET has consideration of only two forms of networks while proposed system offers inclusion of n-number of heterogeneous network. At the same time, the MULMET uses standard queuing model to offer better load balancing. Similar environment is considered in proposed analysis where the size of the queue is investigated as shown in Fig.5. The increasing trend of queue for MULMET is due to conventional usage of the propagation model which incorporates all the data in single queue system. On the other hand, proposed system ProP constructs its own queue length using new extraction attributes. This significant minimizes the dependencies of data piling up in queue resulting in significant control over the average queue length.

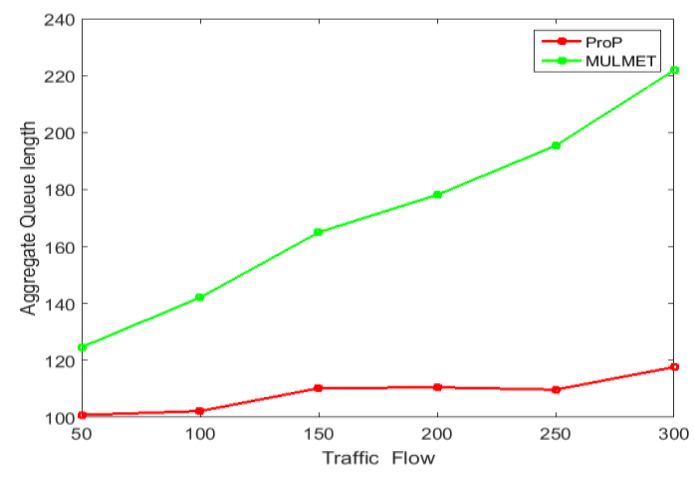

Fig.5. Comparative Analysis of Queue Length

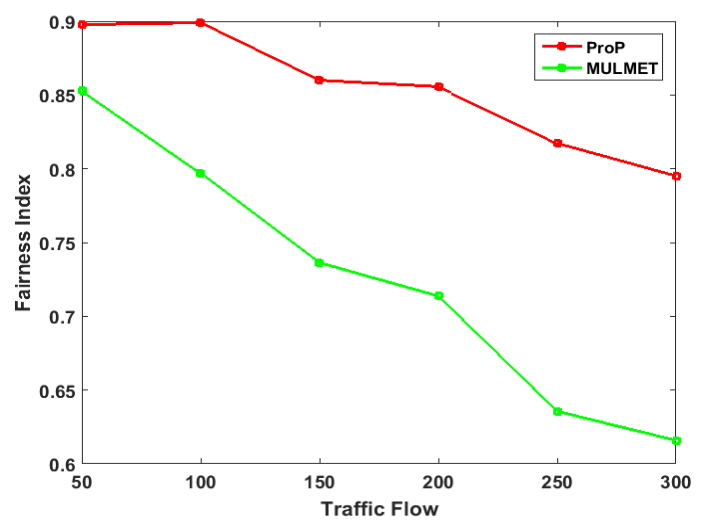

Fig.6. Comparative analysis of Fairness Index

The final analysis of the proposed system uses a new performance parameter called as fairness index in order to understand the betterment in the throughput obtained from the proposed simulation study. Fig.6 shows that there is a declination of the fairness index with increasing traffic flow for both the system. The existing MULMET has the steep declination of the fairness index owing to inclusion of more recursive function while the proposed system offers an efficient allocation of quality of services (e.g. channel capacity) using probability theory. This phenomenon results in efficient sharing of the fairness of the throughput for all the wireless channels which is extremely required for a node to retain a good communication system under foreign network during vertical handover. The approximated computational time for proposed system is 0.6552 seconds while the existing system is 3.4491 seconds. Therefore, the proposed system offers computational efficiency along with resourceefficient communication performance. 


\section{CONCLUSIONS}

An efficient identification of the resource is essentially required when there is present for different number of wireless networks during vertical handover. The proposed system introduces a new module called as extraction attributes that single-handed built its own queue depending upon the present state of the dynamic traffic during vertical handover. Usage of probability theory assists to perform a measureable modeling to ensure better scalability of the developed framework by determining the highest possible performance of the network devices. The system also harnesses the potential of network devices which identifies the necessary amount of resource in order to successfully perform data transmission during the vertical handover mechanism. The study outcome also highlights better throughput, reduced length of queue, and good fairness as compared to existing system.

\section{REFERENCES}

[1] Muntean, Gabriel-Miro, Wireless Multi-Access Environments and Quality of Service Provisioning: Solutions and Application: Solutions and Application, IGI Global, 2012

[2] S. Chen, Y. Shi, B. Hu, Ming Ai, Mobility Management: Principle, Technology and Applications, Springer,2016

[3] Dharma P. Agrawal, Qing-An Zeng, Introduction to Wireless and Mobile Systems, Cengage Learning, 2015

[4] J. L. Burbank, Julia Andrusenko, Jared S. Everett, William T.M. Kasch, Wireless Networking: Understanding Internetworking Challenges, John Wiley \& Sons, 2013

[5] S. G. Glisic, Advanced Wireless Networks: Technology and Business Models, John Wiley \& Sons, 2016

[6] B. U. Kazi and G. Wainer, "Handover enhancement for LTE-Advanced and beyond heterogeneous cellular networks," 2017 International Symposium on Performance Evaluation of Computer and Telecommunication Systems (SPECTS), Seattle, WA, 2017, pp. 1-8.

[7] M. V. Nandkishor and S. Rajashree, "Packet Loss in Handover for Host Based Mobility Management Protocols," 2014 Fourth International Conference on Communication Systems and Network Technologies, Bhopal, 2014, pp. 239-243.

[8] W. Wiharti, I. L. Rimra, Efrizon and I. Novid, "A comparative analysis and survey of handoff for VoIP applications in mobile IPv6 protocols," 2015 2nd International Conference on Information Technology, Computer, and Electrical Engineering (ICITACEE), Semarang, 2015, pp. 464-468

[9] T. Janevski and K. Jakimoski, "Mobility sensitive algorithm for vertical handovers from WiMAX to WLAN," 2012 20th Telecommunications Forum (TELFOR), Belgrade, 2012, pp. 91-94.

[10] L. A. Magagula and S. V. Dlamini, "Enhancement to seamless vertical handover in a micro-mobility domain," 2013 21st International Conference on Software, Telecommunications and Computer Networks - (SoftCOM 2013), Primosten, 2013, pp. 1-5

[11] I. Demydov, M. Seliuchenko, M. Beshley and M. Brych, "Mobility management and vertical handover decision in an always best connected heterogeneous network," The Experience of Designing and Application of CAD Systems in Microelectronics, Lviv, 2015, pp. 103-105

[12] K. B. Ali, F. Zarai, M. S. Obaidat and L. Kamoun,
"Enhanced MIH Architecture-Aware Radio Resource Management Approach in NGWNs," 2015 IEEE International Conference on Data Science and Data Intensive Systems, Sydney, NSW, 2015, pp. 611-616.

[13] J. Suga and R. Tafazolli, "Joint Resource Management with Reinforcement Learning in Heterogeneous Networks," 2013 IEEE 78th Vehicular Technology Conference (VTC Fall), Las Vegas, NV, 2013, pp. 1-5.

[14] H. L. Wang and S. J. Kao, "A vertical handover scheme from WMAN to WLAN by taking into account the maximum available resource," 2011 6th International Conference on Computer Science \& Education (ICCSE), Singapore, 2011, pp. 1373-1378.

[15] A. H. Zahran and C. J. Sreenan, "Extended Handover Keying and Modified IEEE 802.21 Resource Query Approach for Improving Vertical Handoff Performance," 2011 4th IFIP International Conference on New Technologies, Mobility and Security, Paris, 2011, pp. 1-7.

[16] Danni Guo and Xiaobin Li, "An adaptive vertical handover algorithm based on the analytic hierarchy process for heterogeneous networks," 2015 12th International Conference on Fuzzy Systems and Knowledge Discovery (FSKD), Zhangjiajie, 2015, pp. 2059-2064

[17] F. Liu, X. Li and G. Xu, "A MIH and SDN based framework for optimizing vertical handover performance," 2016 International Conference on Progress in Informatics and Computing (PIC), Shanghai, 2016, pp. 555-560.

[18] T. Nguyen-Duc and E. Kamioka, "Feasibility of SDNbased vertical handover between bluetooth and Wi-Fi," 2015 International Conference on Communications, Management and Telecommunications (ComManTel), DaNang, 2015, pp. 24-29.

[19] L. Vuchkovska and K. Jakimoski, "Improvements in the quality of service during vertical handovers between WiMAX and WLAN," 2017 25th Telecommunication Forum (TELFOR), Belgrade, 2017, pp. 1-4.

[20] Z. Gao, C. Chen, Y. Li, B. Wen, L. Huang and Y. Zhao, "A mobility load balancing algorithm based on handover optimization in LTE network," 2015 10th International Conference on Computer Science \& Education (ICCSE), Cambridge, 2015, pp. 611-614.

[21] S. Manfredi, "Cooperative load balancing algorithm in multiple bottleneck networks," 2013 XXIV International Conference on Information, Communication and Automation Technologies (ICAT), Sarajevo, 2013, pp. 1-8.

[22] B. H. Rym and M. Afif, "Load balancing policy for vertical handover between 3G/WiMAX," 2011 4th Joint IFIP Wireless and Mobile Networking Conference (WMNC 2011), Toulouse, 2011, pp. 1-6.

[23] M. Yadollahi, V. T. Vakili, M. Ghaseminajm and A. Jafarian, "Reducing processing delay and ping pong impact of multi attribute decision making handover for heterogeneous wireless networks," 2015 12th International Conference on Telecommunication in Modern Satellite, Cable and Broadcasting Services (TELSIKS), Nis, 2015, pp. 365-368.

[24] GitaMahardhika, Mahamod Ismail, and RosdiadeeNordin, "Vertical Handover Decision Algorithm Using Multicriteria Metrics in Heterogeneous Wireless Network", Hindawi Publishing Corporation, 2015

[25] N. Bagdure and B. Ambudkar, "Reducing Delay during Vertical Handover," 2015 International Conference on Computing Communication Control and Automation, Pune, 2015, pp. 200-204.

[26] Imad El Fachtali,1Rachid Saadane,2 andMohammedElKoutbi, "Vertical Handover Decision Algorithm Using Ants' Colonies for 4G Heterogeneous 
Wireless Networks", Hindawi Publishing Corporation, 2016

[27] S. Kunarak, "A Dynamic Channel Allocation Algorithm Based on Back-Propagation Neural Network for Vertical Handover in HetNets," 2016 UKSim-AMSS 18th International Conference on Computer Modelling and Simulation (UKSim), Cambridge, 2016, pp. 287-292.

[28] K. Shanmugam, "A novel candidate network selection based handover management with fuzzy logic in heterogeneous wireless networks," 2017 4th International Conference on Advanced Computing and Communication Systems (ICACCS), Coimbatore, 2017, pp. 1-6

[29] D. Tian, J. Zhou, H. Qi,, "A Bio-Inspired QoS-Oriented Handover Model in Heterogeneous Wireless Networks", Hindawi Publishing Corporation, 2014

[30] W. Ali, J. Wang, H. Zhu and J. Wang, "An Expedited Predictive Distributed Antenna System Based Handover Scheme for High-Speed Railway," GLOBECOM 2017 2017 IEEE Global Communications Conference, Singapore, 2017, pp. 1-6.

\section{Authors' Profiles}

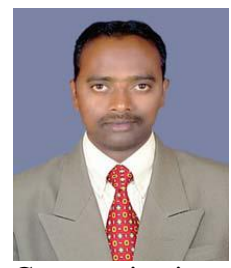

Santosh B. Kumbalavati: (b. July 23, 1986) completed his bachelor degree in Electronics and Communication Engineering in 2008 and completed master degree in Digital Communications in 2010 from Visvesvaraya Technological University (VTU) Belagavi, India. $\mathrm{He}$ is pursuing $\mathrm{PhD}$. in Electronics and Communication Engineering (2012) from Jain University, Bangalore. His areas of interest are Wireless Communication, Mobile Networks, and Computer Communication Networks etc.

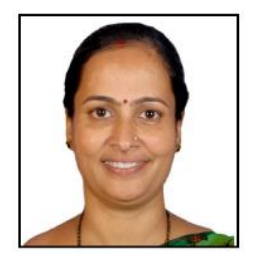

Dr. Jayashree D. Mallapur: (b. Sept. 12, 1969) received $\mathrm{PhD}$ in Electronics and Communication Engineering (2009) from Visvesvaraya Technological University Belagavi, India. She is full time professor in the institute of Basaveshwar Engineering College Bagalkot, affiliated to VTU Belagavi. Her current research interests include Wireless Networks and Fuzzy Applications. She has published more than 47 National/International papers/journals. She also has been the chair person of several conferences.

How to cite this paper: S. B. Kumbalavati, J. D. Mallapur, "Modeling of Seamless Vertical Handover Mechanism using Demanded Resource Identification \& Mapping", International Journal of Information Technology and Computer Science(IJITCS), Vol.10, No.8, pp.31-37, 2018. DOI: 10.5815/ijitcs.2018.08.05 